\title{
Detecção de Treponema pallidum em amostras de sangue fixadas em cartão para diagnóstico da sífilis congênita
}

\author{
Detection of Treponema pallidum in blood samples fixed on card for diagnosis of congenital syphilis \\ Detección de Treponema pallidum en muestras de sangre fijadas en tarjeta para diagnóstico de
} sífilis congénita

Recebido: 06/08/2021 | Revisado: 13/08/2021 | Aceito: 15/08/2021 | Publicado: 17/08/2021

Vera Mileide Trivellato Grassi

ORCID: https://orcid.org/0000-0003-0987-5084

Universidade Luterana do Brasil, Brasil

E-mail: vmgrassi@hotmail.com

Rafaela Caroline Clarinda Melo

ORCID: https://orcid.org/0000-0003-1945-4072

Universidade Federal de Ciências da Saúde de Porto Alegre, Brasil

E-mail:rafaelaccm@gmail.com

Liliane Trivellato Grassi

ORCID: https://orcid.org/0000-0003-0768-943X Universidade Brasil, Brasil

E-mail:lilianegrassi@hotmail.com

Marcia Susana Nunes Silva

ORCID: https://orcid.org/0000-0001-8854-582X DNTech - Desenvolvimento de Novas Tecnologias LTDA, Brasil

E-mail: marcia_susana@hotmail.com

Maria Lucia Rosa Rossetti

ORCID: https://orcid.org/0000-0002-9672-9394 Universidade Luterana do Brasil, Brasil

E-mail: maria.rossetti@ulbra.br

\begin{abstract}
Resumo
A sífilis congênita (SC) é uma doença de transmissão vertical causada pelo Treponema pallidum provocando uma infecção do bebê ainda no útero. O diagnóstico da SC é difícil e tem sido feito por uma combinação de critérios clínicos e laboratoriais, o que torna necessário a busca por novos métodos de diagnóstico para auxiliar a tomada de decisão. Os testes moleculares, apesar de ainda não serem rotina nos laboratórios, estão sendo cada vez mais utilizados. A etapa pré-analítica de coleta da amostra, extração e preservação do DNA é fundamental. O uso de sangue fixado em cartão (SFC) tem sido uma alternativa no diagnóstico de várias doenças pela facilidade na execução da etapa pré-analítica. Nosso trabalho teve como objetivo detectar T. pallidum por reação em cadeia da polimerase (PCR) a partir de SFC de recém-nascidos que tiveram resultado positivo no PCR com a amostra de líquido cefalorraquidiano (LCR). Foram analisadas 82 amostras pareadas (sangue e LCR) de 41 RN na do Hospital da Criança Santo Antônio (HCSA), de Porto Alegre, RS, Brasil, sendo 22 positivas e 19 negativas. A sensibilidade e especificidade foram 63,3\% e $100 \%$, respectivamente. A concordância entre os métodos foi substancial $(\mathrm{K}=0,619)$, mostrando que a PCR diretamente de SFC pode ser promissora, desde que aperfeiçoada sendo uma possibilidade de diagnosticar a SC e facilitar a triagem neonatal.
\end{abstract}

Palavras-chave: Sífilis congênita; PCR; Diagnóstico; Treponema pallidum.

\begin{abstract}
Congenital syphilis (CS) is a vertically transmitted disease caused by Treponema pallidum causing an infection of the baby in the uterus. Diagnosis of CS is difficult and has been made by a combination of clinical and laboratory criteria, which makes it necessary to search for new diagnostic methods to aid decision making. Molecular tests, despite not being routine in laboratories, are being used more and more. The pre-analytical stage of sample collection, DNA extraction and preservation is essential. The use of fixed blood on a card (SFC) has been an alternative in the diagnosis of various diseases due to the ease of carrying out the pre-analytical stage. Our study aimed to detect $\mathrm{T}$. pallidum by polymerase chain reaction (PCR) from CFS of newborns that had a positive PCR result with the cerebrospinal fluid (CSF) sample. . Eighty-two paired samples (blood and CSF) were analyzed from $41 \mathrm{NB}$ at the Hospital da Criança Santo Antônio (HCSA), in Porto Alegre, RS, Brazil, 22 positive and 19 negative. Sensitivity and specificity were $63.3 \%$ and $100 \%$, respectively. The agreement between the methods was substantial $(\mathrm{K}=0.619)$,
\end{abstract}


showing that PCR directly from CFS can be promising, as long as it is improved, it is a possibility to diagnose CS and facilitate neonatal screening.

Keywords: Congenital syphilis; PCR; Diagnosis; Treponema pallidum.

\section{Resumen}

La sífilis congénita (SC) es una enfermedad de transmisión vertical causada por Treponema pallidum que causa una infección del útero en el bebé. El diagnóstico de SC es difícil y se ha realizado mediante una combinación de criterios clínicos y de laboratorio, lo que hace necesaria la búsqueda de nuevos métodos diagnósticos que ayuden a la toma de decisiones. Las pruebas moleculares, a pesar de no ser una rutina en los laboratorios, se utilizan cada vez más. La etapa preanalítica de recolección de muestras, extracción y preservación del ADN es esencial. El uso de sangre fijada en tarjeta (SFC) ha sido una alternativa en el diagnóstico de diversas enfermedades debido a la facilidad para realizar la etapa preanalítica. Nuestro estudio tuvo como objetivo detectar T. pallidum por reacción en cadena de la polimerasa (PCR) de SFC de recién nacidos que tuvieron un resultado de PCR positivo con la muestra de líquido cefalorraquídeo (LCR). Se analizaron 82 muestras pareadas (sangre y LCR) de 41 RN del Hospital da Criança Santo Antônio (HCSA), en Porto Alegre, RS, Brasil, 22 positivas y 19 negativas. La sensibilidad y la especificidad fueron 63,3\% y $100 \%$, respectivamente. La concordancia entre los métodos fue sustancial $(\mathrm{K}=0,619)$, lo que demuestra que la PCR directamente del SFC puede ser prometedora, siempre que se mejore, es una posibilidad para diagnosticar el SC y facilitar el cribado neonatal.

Palabras clave: Sífilis congénita; PCR; Diagnóstico; Treponema pallidum.

\section{Introdução}

A sífilis é uma infecção sexualmente transmissível (IST), grave, infectocontagiosa e sistêmica, causada pelo Treponema pallidum (Macedo et al.,2015).

Em 2016, a ocorrência da sífilis em gestantes no mundo foi de quase um milhão de casos. Entre estes casos, houve 143 mil abortamentos ou mortes fetais, 14 mil mortes neonatais, 41 mil prematuros ou com baixo peso ao nascer, por fim, 109 mil recém-nascidos (RN) infectados desenvolveram complicações clínicas. Nos países onde o investimento em saúde é menor, o acesso aos serviços de saúde também é mais dificil, incluindo os cuidados pré-natais. (WHO, 2020).

Existe uma tendência mundial para redução nas taxas de sífilis congênita (SC) nos últimos anos, mas esta tendência não está sendo observada nas Américas e no Mediterrâneo Oriental, que apresentaram aumento destas taxas. $\mathrm{O}$ aumento observado nas Américas vem ao encontro do aumento no número de casos de sífilis adquirida identificado na população geral desta região nos últimos anos (Ghanem, Ram \& Rice, 2020).

No Brasil, de 1988 a junho de 2018, foram notificados no Sistema de Informações de Agravos de Notificação (Sinan) 188.445 casos de SC em menores de um ano. (Brasil, 2019)

As manifestações clínicas da SC podem surgir logo após o nascimento, ou nos primeiros dois anos de vida. A neurossífilis é observada em $40 \%$ a $60 \%$ das crianças nessa fase podendo evoluir para óbito (Focaccia et al.,2015).

O diagnóstico precoce da SC é importante para um melhor prognóstico. No entanto, o diagnóstico muitas vezes é difícil e não consegue definir com clareza uma infecção ativa, seja pela impossibilidade de cultivo do T. pallidum, ou pela falta de precisão dos testes sorológicos (treponêminos ou não). (Sec. Est. de São Paulo, 2016).

Ao verificarmos as recomendações do CDC e das diretrizes europeias, identifica-se a multiplicidade de situações maternas e nos recém-nascidos que podem ser encontradas na avaliação clínico-laboratorial de casos suspeitos. A multiplicidade de testes a serem solicitados indica que a sensibilidade e a especificidade dos testes se encontram aquém do ideal, e tal fato também se reflete na interpretação sempre relativa dos resultados. É plenamente justificada, portanto, a busca por testes com maiores sensibilidade e especificidade e que possam auxiliar a tomada de decisão clínica. (Janier et al.,2014, Workowski \& Bolan, 2015).

O sequenciamento genético do T. pallidum em 1998 (Weinstock, Hardham, McLeod \& Sodergreen, 1988; Fraser et al.,1988) impulsionou o desenvolvimento de testes moleculares, principalmente os baseados na amplificação por reação em cadeia da polimerase (PCR) para detectar regiões específicas desta bactéria (Fraga et al., 2014). Os dois genes mais utilizado 
para amplificação tem sido o polA (DNA polimerase) e o tpp47, gene que codifica uma proteina de membrana envolvida na sintese da parede celular com sensibilidade e especifidade semelhantes. As amostras mais utilizadas para detecção do $T$. pallidum são liquido cefalorraquidiano (LCR), soro, sangue total e até sangue de cordão umbilical ( Pinilla, Chavarro, Moreno, Navarrete \& Muñoz, 2015; Pinilla, Campos, Duran, Navarrete \& Muñoz, 2018; Theel, Katz \& Pillay, 2020).

O uso de sangue fixado em cartão (SFC) para coletar, transportar e extrair DNA de amostras fixadas tem sido relatado para diagnóstico de várias doenças como a tuberculose, a leishmaniose, sarampo e rubéola (Ali, Bello, Rossetti, Krieger \& Costa, 2020; Mota, Venazzi, Zanzarini, Aristides, Lonardoni \& Silveira, 2020; Bankamp et al.,2019). Este método é ideal para diagnosticar infecções com custos baixos, e volumes mínimos de sangue envolvidos e capacidade de armazenamento e transporte à temperatura ambiente. (Iroh Tam et al.,2018).

No Brasil, esses cartões também são utilizados para coleta de sangue de RN para a triagem neonatal (teste do pezinho) que inclui o diagnóstico de doenças metabólicas, congênitas e infecciosas (Brasil, 2021).

Nosso trabalho abordará a utilidade da coleta de sangue do recém-nascido e este ser fixado em cartão para detecção de DNA de T. pallidum para verificar a presença de sífilis congênita, comparando com o mesmo procedimento em amostras de LCR e testes sorológicos de recém-nascidos de mães com e sem sífilis.

Desta forma o objetivo deste trabalho foi detectar a sífilis congênita através da presença de DNA do T pallidum por PCR em amostras de sangue total fixadas em cartão e comparar com o LCR de recém-nascidos. Avaliar o comportamento da reação em cadeia da polimerase (PCR) para a deteç̧ão do genoma de T. pallidum nas diferentes amostras de recém-nascidos em diferentes situações de risco para sífilis congênita, determinar a concordância entre as duas técnicas e determinar a sensibilidade e especificidade, do método de PCR para diagnóstico de sífilis congênita.

\section{Metodologia}

Este é um estudo experimental e transversal. Foram coletadas 41 amostras de sangue total de recém-nascidos, sendo 22 amostras de recém-nascidos de mães com diagnóstico de sífilis, (segundo os critérios do CDC e das diretrizes europeias (Janier et al.,2014; Workowski et al.,2015) e com PCR de LCR positivo, e 19 amostras de sangue total de recém-nascidos, com indicação de coleta de sangue e líquor para investigação de sepse neonatal precoce, ou TORCH (Toxoplasmose, Rubéola, Citomegalovírus, e vírus Herpes simples), de mães sem suspeita de sífilis (usados como controle), na maternidade do Hospital da Criança Santo Antônio, de Porto Alegre, Rio Grande do Sul. O diagnóstico de sífilis nas mães foi realizado por testes laboratoriais sorológicos (RPR), e clínicos realizados por equipe de profissionais do próprio hospital. Todos os RN fizeram o teste RPR (rapid plasma reagin) no sangue.

As amostras foram enviadas para o laboratório de biologia molecular da Ulbra Canoas, onde foram realizados de agosto de 2019 a junho de 2020 os testes de PCR.

O projeto foi aprovado pela CEP da Universidade Federal de Ciências da Saúde de Porto Alegre (2.547.348 ) e do HCSA (3.432.633).

\section{Extração de DNA}

Foi distribuído $40 \mu \mathrm{l}$ de sangue no cartão FTA Elute ${ }^{\circledR}$ (Whatman ${ }^{\mathrm{TM}}$ ) e secado à temperatura ambiente. A extração de DNA do cartão foi realizada com dois discos de seis mm removidos do cartão (conforme instruções do fabricante).

O DNA de LCR foi extraído com Kit PureLink® Genomic (Invitrogen®, Thermo Fisher Scientific, EUA), conforme instruções do fabricante. 


\section{Detecção do T. pallidum por PCR}

A detecção de T. pallidum foi realizada pela técnica de PCR convencional utilizando os primers KO3 (5' GAAGTTTGTCCCAGTTGCGGTT-3') e KO4 (5'CAGAGCCATCAGCCCTTTTCA 3') que amplificam um segmento de $260 \mathrm{pb}$ do gene tpp 47 conforme descrito por Palmer \& Higgins \& Herring \& Kingston, 2003.

A amplificação do DNA foi realizada com uma mistura com tampão 10X, 1,5 mM MgCl2, $200 \mu \mathrm{M}$ de cada dNTPs, 25 pmol de cada primer e 1,25 U da enzima Taq platinium DNA polimerase. Foi feita uma desnaturação inicial a $95^{\circ} \mathrm{C}$ por 2 minutos, seguida de uma desnaturação a $95^{\circ} \mathrm{C}$ por 20 segundos, $62^{\circ} \mathrm{C}$ por 20 segundos e $72^{\circ} \mathrm{C}$ por 20 segundos, durante 35 ciclos, e uma extensão final a $72^{\circ} \mathrm{C}$ por 5 minutos. A amplificação em aparelho termociclador MJ Research PTC 96.

O resultado da PCR foi analisado por eletroforese em gel de agarose $2 \%$ contendo 0,05\% de brometo de etídio e visualizado sob luz ultravioleta.

O controle positivo foi DNA de T. pallidum e como controle negativo, uma reação somente com a mistura de reagentes, livres de DNA.

\section{RPR}

O teste de RPR foi realizado nos RN foram conforme recomendado no guideline europeu (Janier et al.,2014)

\section{Análises Estatísticas}

Os dados foram alocados em uma base de dados no programa Microsoft Excel, e foram processados e analisados pelo programa Statistical Package for the Social Sciences (SPSS) v21. Para avaliar a concordância entre os resultados de PCR para detecção do patógeno, realizados pelas diferentes extrações, foi calculado o índice Kappa (em pares), onde a força de concordância Kappa $(\mathrm{K})$ foi interpretada da seguinte maneira: $\mathrm{K}=<0,00$ (insignificante), $\mathrm{K}=0,00-0,19$ (ruim), $\mathrm{K}=0,20-0,39$ (fraca), $\mathrm{K}=0,40-0,59$ (moderada), $\mathrm{K}=0,60-0,79$ (importante), $\mathrm{K}=0,80-0,99$ (quase perfeita) e $\mathrm{K}=1,00$ (perfeita) (Iared et al.,2018).

\section{Resultados}

Os 22/41 RN considerados positivos para a infecção por T. pallidum, quando o PCR foi realizado do SFC foi possível amplificar 14/22 (63,6\%) e em oito não houve amplificação. Os PCRs de SFC dos RN controles não amplificaram T. pallidum (Tabela 1). A sensibilidade foi de 63,3\% e a especificidade foi de $100 \%$ do PCR com SFC.

Tabela 1: Resultados de PCR de LCR e do SFC para Treponema pallidum.

\begin{tabular}{llllll}
\hline & Positivo $(\mathrm{n}=22)$ & Negativo $(\mathrm{n}=19)$ & Sensibilidade & Especificidade & Kappa \\
\hline SFC $(\mathrm{n}=41)$ & & & $63,3 \%$ & $100 \%$ & 0,619 \\
$\begin{array}{l}\text { Positivo } \\
\text { Negativo }\end{array}$ & 14 & 0 & & & \\
\hline
\end{tabular}

LCR $(n=41)$. Fonte: Autores.

A concordância entre os métodos de PCR (LCR e SFC) foi considerada substancial (Kappa =0,619). Os resultados da comparação do PCR com o teste RPR estão na tabela 2. 
Tabela 2: Comparação entre os resultados de PCR/LCR, PCR/Sangue e RPR.

\begin{tabular}{llllll}
\hline & Positivo (n=15) & Negativo (n=26) & Sensibilidade & Especificidade & Kappa \\
\hline $\begin{array}{l}\text { PCR/LCR (n=41) } \\
\text { Positivo }\end{array}$ & 15 & 7 & $100 \%$ & $73,0 \%$ & 0,66 \\
Negativo & 0 & 19 & & & \\
\hline $\begin{array}{l}\text { PCR/Sangue } \\
\text { (n=41) }\end{array}$ & & & $66,6 \%$ & $84,0 \%$ & 0,52 \\
Positivo & 10 & 4 & & & \\
Negativo & 5 & 22 & & & \\
\hline
\end{tabular}

RPR ( $\mathrm{n}=41)$. Fonte: Autores.

\section{Discussão}

A possibilidade de detecção de T. pallidum por PCR confirmando que este pode auxiliar na definição de infecção ativa, tem sido relatado (Castro et al.,2016; Theel et al.,2020). Nosso estudo analisou a deteç̧ão de T. pallidum por PCR para o diagnóstico da SC em RN a partir de SFC e comparou com RN com suspeita de SC e com PCR de LCR positivo (padrão de referência) e controles. A sensibilidade encontrada foi de $63 \%$ e está dentro do que tem sido relatado (14\% a 63\%) em PCRs com sangue e derivados (soro, plasma) independentemente do estágio clínico em que os pacientes se encontravam (Wang et al.,2018). A concordância entre os testes foi de $K=0,619$ que é considerada substancial, mostrando que o SFC pode ser usado para detectar o T.pallidum. No entanto, a especificidade do PCR foi de $100 \%$, o que o torna importante pois possibilita a exclusão de um falso positivo como caso de SC.

Quando comparamos os resultados de ambos os PCR com o de RPR observamos que a sensibilidade foi de $100 \%$, e $66,6 \%$ e a especificidade de 73,0\% e 84,0\% no LCR e no SFC, respectivamente. A concordância foi avaliada como substancial $(\mathrm{K}=0,665)$ no LCR e moderada $(\mathrm{K}=0,52)$ no SFC. Quando consideramos o RPR como referência, os resultados falso positivos sugerem que pode não ter ainda transferência de anticorpos maternos ao feto, mas que a bactéria atravessou a barreira placentária. Já nos resultados falso negativos sugerem a transferência de anticorpos maternos, pois RN ainda não tem anticorpos próprios, mas que não houve transferência da bactéria, ou podem ser explicado por uma possível carga bacteriana baixa circulando no sangue, ou ainda pela não homogeneidade com que o DNA presente no sangue se espalha no cartão, assim algumas amostras só se mostraram positiva nas amostras de LCR (Michelow et al.,2002; Castro et al.,2016; Mota et al.,2021) .

O teste Venereal Disease Research Laboratory (VDRL), teste amplamente utilizado para o diagnóstico de sífilis, possui uma sensibilidade em amostras de LCR de RN em torno de 50\% e sua significância ainda não está clara, devido muitos falsos negativos e positivos, relacionados a janela imunológica e anticorpos maternos que cruzam a placenta (Michelow et al.,2002; Woods et al.,2005; Brasil, 2016). Assim apesar da sensibilidade encontrada no nosso estudo nas amostras de cartão ser de 63,3\% ainda é melhor que os métodos atualmente utilizados.

Não foram encontrados estudos com SFC para detecção do T.pallidum, mas um tipo de papel filtro o cartão de Guthrie (teste do pézinho) já é utilizado com sucesso na triagem neonatal de várias doenças e o cartão Whatman (FTA- elute ou Classic) já faz parte da detecção de outros agentes biológicos (Bankamp et al.,2019; Ali et al.,2020; Mota et al.,2021).

\section{Conclusão}

Conclui-se com nosso estudo que apesar da sensibilidade do SFC ter sido de apenas 63,3\%e a especificidade de 100\%, mostrou que este pode ser um método de diagnóstico de SC em RN. O método de PCR diretamente de SFC é uma técnica que se aperfeiçoada pode ser muito promissora para a avaliação de casos suspeitos e proporcionar uma melhora no 
diagnóstico desta doença tão negligenciada, mas ainda há a necessidade de continuar os estudos sobre a técnica e a importância do uso, pois coletar amostras de LCR de todos os RN é muito invasivo e as amostras de sangue fixadas em cartão é de fácil coleta e transporte. Por fim, este artigo lança luz sobre um tema muitas vezes negligenciado, que ainda tem poucos dados publicados e apresenta uma visão geral das variáveis clínicas de interesse.

\section{Agradecimentos}

O presente trabalho foi realizado com o apoio da Coordenação de Aperfeiçoamento de Pessoal de Nível SuperiorBrasil (CAPES) código de financiamento 001.

\section{Referências}

Ali, N., Bello, G. L., Rossetti, M. L. R., Krieger, M. A., \& Costa, A. D. T. (2020). Demonstração de um protocolo rápido e fácil de amostra para resposta para rastreamento de tuberculose em ambientes de ponto de cuidado: Uma prova de estudo conceitual. PLoS ONE 15(12): e0242408. https://doi.org/10.1371/journal.pone.0242408

Bankamp, B., Sein, C., Pukuta- Simbu, E., Anderson, R., Abernathy, E., Chen, M-H., Muyembe-Tamfum, J-J., Wannemuehler, K. A., Waku-Kouomou, D., Lopareva, E. N., Icenogle, J. P., Rota, P. A., \& Goodson, J. L. (2019). Use of FTA cards to transport throat swabs and oral fluid samples for molecular detection and genotyping of measles and rubella viruses. Journal of Clinical Microbiology 57:e00048-19. https://doi.org/10.1128/JCM .00048-19

Brasil, Boletim Epidemiológico da Sífilis. Ministério da Saúde, 2019

Brasil, Governo do. (2021). Teste do Pezinho será ampliado e detectará até 50 novas doenças.

Brasil. Ministério da Saúde, Secretaria de Vigilância em Saúde, Departamento de Vigilância, Prevenção e Controle das Doenças Sexualmente Transmissíveis, Aids e Hepatites Virais. Manual Técnico para Diagnóstico da Sífilis: Ministério da Saúde, 2016.

Castro, R., Aguas, M. J., Batista, T., Araujo, C., Mansinho, K., \& Pereira, F. L. M. (2016). Detection of Treponema pallidum Sp. Pallidum DNA in Cerebrospinal Fluid (CSF) by to PCR Techniques. Journal of Clinical Laboratory Analysis. 30: 628-632. DOI 10.1002/jcla.21913

Focaccia R. Tratado de infectologia. (5a ed.), editora Atheneu; 2015.

Fraga, D., Muller, A. L., Czykiel, M. S., De Armas, Y., Wissmann, G., \& Goldani, L. Z. (2014). Detection of Treponema pallidum by semi-nested PCR in the cerebrospinal fluid of asymptomatic HIV-infected patients with latent syphilis. Clinical Laboratory. 60(12):2051-2054. 10.7754/clin.lab.2014.140605

Fraser, C. M., Norris, S. J., Weinstock, G. M., White, O., Sutton, G. G., Dodson, R., \& Sodergreen, E. (1988). Complete genome sequence of Treponema pallidum, the syphilis spirochete. Science. 281, 375-388. 10.1126/science.281.5375.375

Ghanem, K. G., Ram, S., \& Rice, P. A. (2020). The Modern Epidemic of Syphilis. The New England Journal of Medicine. 382(9):845-854. 10.1056/NEJMra1901593.

Iared, W., Puchnick, A., Bancovsky, E., Bettini, P. R., Vedolin, L. M., \& Chammas, M. C. (2018). Reproducibility of a quantitative system for assessing the quality of diagnostic ultrasound. Radiologia Brasileira. Mai/Jun;51(3):172-177. 10.1590/0100-3984.2017.0021.

Iroh Tam, P-Y., Hernandez-Alvarado, N., Schleiss, M. R., Hassan-Hanga, F., Onuchukwu, C., Umoru, D., \& Obaro, S. K. (2016) Molecular Detection of Streptococcus pneumoniae on Dried Blood Spots from Febrile Nigerian Children Compared to Culture. PLoS ONE 11(3): e0152253. https://doi.org/10.1371/journal.pone.

Janier, M., Hegyi, V., Dupin, N., Unemo, M., Triplica, G. S., Potocnik, M., Frances, P., \& Patel, R. (2014). European guideline on the management of syphilis. Journal of the European Academy of Dermatology and Venereology. 28(12):1581-1593. 10.1111/jdv.12734

Korenromp, E. L., Rowley, J., Alonso, M., Mello, M. B., Wijesooriya, N. S., Mahiané, S. G., Ishikawa, N., Le, L. V., Newman-Owiredo, M., Magalkerke, N., Newman, L., Broutet, N., \& Taylor, M. M. (2019) Global burden of maternal and congenital syphilis and associated adverse birth outcomes, Estimates for 2016 and progress since 2012. PLoS ONE 14(2): e0211720. https://doi.org/10.1371/journal.pone.0211720

Macedo, E. M. (2005) Há 100 anos, a descoberta do Treponema pallidum. Anais Brasileiros de Dermatologia. 80, 547-548. https://doi.org/10.1590/S036505962005000600017

Michelow, I. C., Wendel, G. D., Norgard, M. V., Zeray, F., Leos, N. K., Alsaadi, R., \& Sánches, P. J. (2002). Central Nervous System Infection in Congenital Syphilis. The New England Journal of Medicine. 346(23):1792-8. 10.1056/NEJMoa012684.

Mota, C. A., Venazzi, E. A. S., Zanzarini, P. D., Aristides, S. M. A., Lonardoni, M. V. C., \& Silveira, T. G. V. (2021). Filter paper performance in PCR for cutaneous leishmaniasis diagnosis. Revista da Sociedade Brasileira de Medicina Tropical. 54. https://doi.org/10.1590/0037-8682-0047-2020

Palmer, H. M., Higgins, S. P., Herring, A. J., \& Kingston, M. A. (2003). Use of PCR in the diagnosisof early syphilis in the inited Kingdom. Sexually Transmitted Infections. 79:479-83. DOI.org/10.1136/sti.79.6.479

Pinilla, G., Campos, L., Durán, A., Navarrete, J., \& Muñoz, L. (2018). Detección de Treponema pallidum subespecie pallidum para el diagnóstico de sífilis congénita mediante reacción en cadena de la polimerasa anidada. Biomedica. 38(1):128-35. 10.7705/biomedica.v38i0.3740. PMID: 29676865. 
Research, Society and Development, v. 10, n. 10, e511101019151, 2021

(CC BY 4.0) | ISSN 2525-3409 | DOI: http://dx.doi.org/10.33448/rsd-v10i10.19151

Pinilla, G. B., Chavarro, B. P., Moreno, M. A., Navarrete, J. O., \& Muñoz, L. M. (2015). Determinación de los genes, $16 S A D N r$, polA, y TpN47, em la detección de Treponema pallidum subsp. pallidum para el diagnóstico de sífilis congénita. NOVA. 2015; 13 (23): 17-25. https://doi.org/10.22490/24629448.1713

Secretaria do estado de São Paulo. Guia de bolso- Manejo da sífilis em gestante e sífilis congênita. 2016. (2a ed.).

Theel, E. S., Katz, S. S., \& Pillay, A. (2020). Molecular and Direct Detection Tests for Treponema pallidum Subspecies pallidum: A Review of the Literature, 1964-2017. Clinical Infectious Diseases. 71(Supplement_1):S4-S12. 10.1093/cid/ciaa176

Wang, C., Cheng, Y., Liu, B., Wang, Y., Gong, W., Qian, Y., Guan, Z., Lu, H., Gu, X., Shi, M., \& Zhou, P. (2018). Sensitive detection of Treponema pallidum DNA from the whole blood of patients with syphilis by the nested PCR assay. Emerging Microbes Infections, 7:1, 1-7, 10.1038/s41426-018-0085-2 t.

Weinstock, G. M., Hardham, J. M., McLeod, M. P., Sodergren, E. J., \& Norris, S. J. (1988). The genome of Treponema pallidum: New light on the agent of syphilis. FEMS Microbiol Rev. 1998;22(4):323-332.

Woods C. R. Syphilis in children: congenital and acquired. Seminars in Pediatric Infectious Diseases. 16(4):245-257. doi.org/10.1053/j.spid.2005.06.005

Workowski, K. A., \& Bolan, G. A. (2015). Centers for Disease Control and Prevention. Sexually transmitted diseases treatment guidelines, 2015. Morbidity and Mortality Weekly Report. Recommendations and Reports. 64(RR-03):1-137. Erratum in: MMWR Recomm Rep. 64(33):924.

World Health Organization Global adult estimates of chlamydia, gonorrhoea, trichomoniasis and syphilis including maternal and congenital syphilis, 2016, WHO; 2020, 76p. 